\title{
Application of M00 Techniques for the Time-Cost Trade off Problems in Civil Engineering
}

\author{
Tayfun DEDE* and Soufiane KAMİL \\ Department of Civil Engineering, Karadeniz Technical University, Turkey
}

*Corresponding author: Tayfun DEDE, Department of Civil Engineering, Karadeniz Technical University, Turkey.

Received Date: December 23, 2018

Published Date: January 18, 2019

\section{Editorial}

Multi-objective optimization (MO0) defined as multi objective programing used specially for mathematical optimization problems involving more than one target to minimize or maximize. Multiobjective optimization is applied to find set of solutions as close as possible to the optimal front. In addition, MOO is used in different science field as economic, logistics and engineering. Construction industry is one of the fields that benefit the most from multiobjective optimization, because the optimization is used while designing by optimizing different parameters such as area, material, energy. In the construction management the time, cost, quality and resources can be as an objective function to optimize the total construction activities. Also, in one hand the aim of minimizing the material and the cost and in the second hand the aim of maximizing the stability, stiffness and strength engineers are forced to use a Multi-Objective optimization. That why nowadays we find many researches according to Multi-objective optimization.

Time-Cost Trade-off is used to determine the most effective way to decrease the overall duration of project and increase the targeted budget. If project manager needs to shorten an activity, he has to do it in the most cost-effective way. Since there is a tradeoff relation between time and cost it is difficult to prognosticate whether the total cost will increase or decrease by compressing the schedule. For this reason, completing a project on the scheduled time also on or under the targeted budget is an important aim for the manager; to reach it the use of Multi-objective optimization in different techniques is needed. Modified adaptive weight approach is one of the techniques used to solve a multi-objective optimization problem. This approach simply assigns weights to each objective function and combines them into a single objective function. It is the approach which has got the simplest formulation and easy to be implemented. In spite of being simple one, is able to achieve optimum or near optimum solutions as no further interaction with the decision-makers is needed. Modified adaptive weight approach (MAWA) proposed by Zheng et al, 2004; to identify adaptive weight for each objective, MAWA benefits the information from the existing set of solutions [1].

As it is obvious, nowadays, instead of modified adaptive weight approach (MAWA) approach, this non-dominating sorting (NDS) superior approach is extensively being acknowledged in unraveling the different benchmark optimization TCTP problems. In contrast to MAWA approach, there is no unique solution provided by NDS approach, but Pareto front solutions are produced and selected by comparing two solutions to each other. This NDS approach seeks the satisfactory solution from the non-dominated solutions depending on the experience and knowledge of decision-makers. The domination concept defined as: design A dominates design $B$ if it is better in at least one criterion and not worse in all other objectives Deb et al. [2]. The process of sorting designs variables based on dominance is called non-dominated sorting (NDS). At any phase in an optimization run, a population or repository of "current" designs is kept up. At each progression, every feasible design that is not dominated by some other designs in the population (or archive) is given the rank of 1 . These are the just non-dominated designs in the population. At that point, these designs are adroitly expelled from the repository, and the rest of the designs are judged for domination. Those that are not dominated by any of the rest of the designs are given the rank of 2 . The method is repeated, repositioning the rest of the designs after eliminating non-dominated designs, to build up ranks 3,4 , and so on. As the run progresses, new designs will dominate and replace other designs on a series of local Pareto fronts. The result will regularly be a combination of variables that are not overwhelmed by any other designs and converge towards the Pareto front. From this bunch of designs, one can pick up the design that best suits the present requirements or those that move towards hunting. 
As motioned before multi-objective optimization is used in different researches some examples can be given as following:

Time-Cost-Quality Trade-off Software by using Simplified Genetic Algorithm for Typical repetitive Construction Projects by Refaat et al. [3]. Construction materials-based Methodology for Time-Cost-Quality Trade-off Problems by Kazaz et al. [4] A review on building energy efficient design optimization rom the perspective of architects by Xing et al. [5].

\section{Acknowledgment}

None.

\section{Conflict of Interest}

No conflict of interest.

\section{References}

1. Zheng DXM, Thomas Ng S, Kumaraswamy MM (2004) Applying a genetic algorithm based multi objective approach for time-cost optimization. Journal of Construction Engineering and Management 130(2): 168 - 176.

2. Deb K, Pratab A, Agrawal S, Meyarivan T (2002) A fast and elitist multiobjective genetic algorithm. NSGA-II. IEEE Trans, Evolution of Computing 6(2): 182- 197.

3. Refaat H, Abd El R, Ahmed MD, Sherif MH, Remon FA (2010) Time-CostQuality Trade-off Software by using Simplified Genetic Algorithm for Typical repetitive Construction Projects. International Journal of Civil and Environmental Engineering 4(1): 22-31.

4. Kazaz A, Ulubeyli S, Bayram Er, Acıkara T (2016) Construction materialsbased Methodology for Time-Cost-Quality Trade-off Problems, Procedia Engineering 164: 35 - 41.

5. Xing S, Zhichao T, Wenqiang C, Binghui S, Xing J (2016) A review on building energy efficient design optimization rom the perspective of architects, Renewable and Sustainable Energy Reviews 65: 872-884. 